\title{
Effect of anti-inflammatory drugs on sulphated glycosaminoglycan synthesis in aged human articular cartilage
}

\author{
LAURA S. McKENZIE, B. A. HORSBURGH, PETER GHOSH, AND T. K. F. TAYLOR \\ From the Raymond Purves Research Laboratories, University of Sydney, The Royal North Shore Hospital of \\ Sydney, St. Leonards, N.S.W. 2065, Australia
}

\begin{abstract}
McKenzie, L. S., Horsburgh, B. A., Ghosh, P., and Taylor, T. K. F. (1976). Annals of the Rheumatic Diseases, 35, 487-497. Effect of anti-inflammatory drugs on sulphated glycosaminoglycan synthesis in aged human articular cartilage. The anti-inflammatory drugs, sodium salicylate, indomethacin, hydrocortisone, ibuprofen, and flurbiprofen, were examined for their effects on sulphated glycosaminoglycan synthesis in aged human cartilage in vitro. Cartilage was obtained from femoral heads removed during surgery and drug effects were found to vary significantly from one head to another. Statistical analysis of the results showed that sodium salicylate exhibits concentration-dependent inhibition of glycosaminoglycan synthesis over the concentration range used. Indomethacin, hydrocortisone, and ibuprofen, at concentrations comparable to those attained in man, caused a statistically significant depression of sulphated glycosaminoglycan synthesis in cartilage from some femoral heads but not others, reflecting the variable response of human articular cartilage to anti-inflammatory drugs. Sodium salicylate and indomethacin at higher doses produced significant $(P<0.005)$ inhibition of sulphated glycosaminoglycan synthesis in all femoral heads studied. The results for flurbiprofen were less conclusive; this compound appears not to inhibit glycosaminoglycan synthesis over the concentration range used.
\end{abstract}

An early event in osteoarthrosis is proteoglycan loss from articular cartilage (Mankin, 1974; McDevitt, 1973; Lust and Pronsky, 1972; Bjelle and others, 1972; Mankin and Lipiello, 1970, 1971; Bollet and Nance, 1966; Bollet and others, 1963; Matthews, 1953). Proteoglycans impart resilience and molecular exclusion properties to cartilage and depletion can result in fibrillation and impaired joint function (Radin and others, 1975; Bullough, Goodfellow, and O'Connor, 1973; Freeman, 1973; Kempson and others, 1970). In all but severe cases of osteoarthrosis the chondrocyte response to proteoglycan depletion results in an increase in glycosaminoglycan synthesis although the magnitude of this response is debatable (Maroudas, 1975; Mankin and others, 1971). An increase in ${ }^{35} \mathrm{SO}_{4}$ uptake by osteoarthrotic cartilage chondrocytes as compared to normal chondrocytes was observed autoradiographically as early as 1960 by Collins and McElligott. In the later stages of the disease, when severe damage to the cartilage has

Accepted for publication April 25, 1976. Correspondence to Dr. P. Ghosh. occurred, reduced rates of glycosaminoglycan synthesis and chondrocyte death have been reported (Mankin and others, 1971; Mankin and Lipiello, 1970; Collins and McElligott, 1960).

In animal tissues, including cartilage, antiinflammatory drugs have been reported to inhibit glycosaminoglycan synthesis, both in vivo and in vitro (Bostrom, Bersten, and Whitehouse, 1964; Whitehouse and Bostrom, 1962). These agents have also been shown to inhibit the synthesis and release of glycosaminoglycans from cultured fibroblasts (Karzel and Domenjoz, 1969; Kalbhen, Karzel, and Domenjoz, 1967). Whitehouse (1965) noted that a close correlation exists between anti-inflammatory activity and the inhibition of glycosaminoglycan synthesis in such experimental systems.

Osteoarthrosis principally affects the older age groups. In some respects aged human cartilage differs both chemically and metabolically from young animal cartilage (Maroudas, 1975; McDevitt, 1973; Benmaman, Ludoweig, and Anderson, 1969). Therefore, we have examined the effects of some of 
the more commonly prescribed anti-inflammatory drugs on the synthesis of sulphated glycosaminoglycans in aged human cartilage, under rigidly controlled culture conditions. Particularly notable was the highly variable response to the drugs of human cartilage from different individuals, highlighting the need for exacting statistical analysis of the results.

\section{Materials and methods}

ANTI-INFLAMMATORY DRUGS

The following drugs were obtained in pure form from the manufacturers: indomethacin (Merck, Sharp and Dohme, Rahway, N.J.), sodium salicylate (Nelson Laboratories Pty., Sydney, Australia), ibuprofen and flurbiprofen (Boots, Nottingham, England), hydrocortisone sodium succinate as an injectable preparation (Upjohn Pty., Rydalmere, Australia).

SAMPLING OF ARTICULAR CARTILAGE

Human articular cartilage was obtained from femoral heads removed surgically after subcapital fracture. The ages of the patients ranged from 63 to 83 years. A special tool, holding four parallel razor blades spaced $1 \mathrm{~mm}$ apart, was used to cut sections of cartilage $1 \mathrm{~mm}$ wide perpendicular to the articular surface. Great care was taken to sample the full depth of articular cartilage since glycosaminoglycan distribution and metabolism is reported to vary with distance from the articular surface (Jones and Lemperg, 1975; Maroudas, 1975; Stockwell and Scott, 1967). For each femoral head, cartilage was sampled from the superior surface which is generally considered to be a weight-bearing area (Maroudas, Evans, and Almeida, 1973). Each section of cartilage was divided into full-depth slices, $2-3 \mathrm{~mm}$ long, for culturing.

\section{CULTURE METHODS}

Explants were set up in culture within 2 hours of surgical removal and were maintained in organ culture using a modification of the method of Trowell (1959). Explants were supported on stainless steel grids, bathed in culture medium $37^{\circ}$, in a humidified atmosphere of $5 \% \mathrm{CO}_{2}$ in air. The culture medium used was Dulbecco Modified Eagle's Medium supplemented with $10 \%$ fetal calf serum (Commonwealth Serum Laboratories). The serum was found to be essential for maintaining a high level of viability for several days. Under these conditions incorporation of ${ }^{3} \mathrm{H}$-uridine into RNA, and ${ }^{35} \mathrm{SO}_{4}$ into glycosaminoglycans (Curran and Gibson, 1956) showed no reduction for at least 3 days, but all experiments were performed within 2 days. The culture methodology will be fully described elsewhere.

MEASUREMENT OF RADIOSULPHATE UPTAKE INTO SULPHATED GLYCOSAMINOGLYCANS

Synthesis of sulphated glycosaminoglycans was measured by the specific incorporation of ${ }^{35} \mathrm{SO}_{4}$ (Radiochemical Centre, Amersham, England) into the glycosaminoglycan fractions of articular cartilage, rather than uptake into whole tissue. When cartilage slices were incubated with ${ }^{35} \mathrm{SO}_{4}$, considerable nonspecific binding could be measured even in controls incubated at $0^{\circ}$. The majority of unbound ${ }^{35} \mathrm{SO}_{4}$ was removed by washing each slice for $4 \hat{\Phi}$ minutes in four $5 \mathrm{ml}$ changes of ice-cold physiologicat saline containing nonradioactive sulphate as a chaser $\overline{\text {. }}$. Each slice was dried in acetone and alcohol, then under. vacuum at $40^{\circ}$, and weighed. Dry weights were found tê? be more accurate and reproducible than wet weigh $\Phi$ determinations. Slices were solubilized by overnigh $\bar{\Phi}$ digestion at $65^{\circ}$ with crystalline papain (Merck) (1.5 mg $\mathrm{ml}$ in $0.1 \mathrm{~mol} / 1$ acetate buffer $\mathrm{pH} \mathrm{5.6}$, containing 0.05 mol/1 EDTA and $0.005 \mathrm{~mol} / 1$ cysteine). Papain digests were dialysed overnight against water and analysed fơ uronic acid by the method of Bitter and Muir (1962), an for radioactivity using standard scintillation counting techniques. Incorporation of ${ }^{35} \mathrm{SO}_{4}$ into glycosaminogly $\overrightarrow{\tilde{z}}$ cans was expressed as $\mathrm{cpm} / \mu \mathrm{g}$ uronic acid.

\section{ANTI-INFLAMMATORY DRUG STUDIES}

After cartilage slices had been allowed to stabilize ir culture for 1 day, the effects of anti-inflammatory drugs on sulphate incorporation into glycosaminoglycans were్ studied by incubating cartilage slices in medium cono taining the drug and ${ }^{35} \mathrm{SO}_{4}$ at $40 \mu \mathrm{Ci} / \mathrm{ml}$. Each drug was studied at three concentrations whose effects on sulphate incorporation were compared to controls with no added drug. The concentrations used covered the range of therapeutic blood plasma levels reported for the drugs examined (Mills and others, 1973; Chalmers and others 1972; Domenjoz, 1971; Karzel and Domenjoz, 1969;Hucker and others, 1966; Holt and Hawkins, 19\$6 However, indomethacin was also examined at highep concentrations. The effect of the drugs was measuresid over 6 hours, triplicate slices of cartilage being remowed from culture at hourly intervals for each drug concentration and the control, making a total of 72 slices for each drug studied. For these experiments, cartilage slices wero removed from the stainless steel grids to Falcon Micro® test II tissue culture plates (Falcon Plastics, Los Angeles $\overrightarrow{\vec{r}}$ Calif.), which consist of 96 identical wells. One cartilage slice was incubated in each well in $0.25 \mathrm{ml}$ medium containing ${ }^{35} \mathrm{SO}_{4}$ and the anti-inflammatory drug? Incubations were terminated by transferring slices into $5 \mathrm{ml}$ ice-cold saline. All drugs dissolved freely in culture medium except indomethacin, which, however, was soluble in medium at approximately $\mathrm{pH} 9.0$, and remained in solution when the $\mathrm{pH}$ was readjusted to neu-i trality.

STATISTICAL ANALYSIS OF RESULTS

The results of each drug experiment, using cartilage from $D$ a single femoral head, were analysed by a two-factor (fixed model) analysis of variance (Snedecor and Cochran, 1967). This type of analysis determines the significance? of the effect of the drug in question on the synthesis of sulphated glycosaminoglycans over the time range studiedNิ at different drug concentrations. Since each drug was ${ }^{\omega}$ tested using cartilage from more than one femoral head, the significance of the differences between the responses of different femoral heads was determined using a threefactor (mixed value) analysis of variance.

Bartlett's test showed significant heterogeneity of $\square$ variances $(P<0.05)$ and all data were transformed to natural logarithms to stabilize variances. The results obtained using the statistical methods described are $\frac{{ }_{1}}{\mathbb{D}}$ shown in Figs. 1-6 and summarized in Tables I-VI. 


\section{Results}

\section{INDOMETHACIN}

The effect of indomethacin over a concentration range of $0.02-0.005 \mathrm{mmol} / 1$ was studied using cartilage from two femoral heads (FH1 and FH2). The effect of the drug on sulphated glycosaminoglycan synthesis differed for the two specimens. One (FH1) showed a significant inhibition of glycosaminoglycan synthesis $(P<0.005)$ with increasing indomethacin concentration. The other (FH2) showed no significant response to the drug (Fig. 1; Table I). When the results for the two femoral heads were analysed together the difference between them was significant $(P<0.05)$. At the higher concentration range (1.0-0.1 mmol/l), indomethacin was examined for its effect on glycosaminoglycan synthesis using cartilage sampled from three femoral heads (FH3, FH4, and FH5). In each instance a significant depression of sulphated glycosaminoglycan synthesis $(P<0.005)$ was observed with increasing indomethacin concentration (Fig. 2; Table II). When the results for the three femoral heads were analysed together the difference between the individual femoral heads was highly significant $(\mathrm{P}<0.005)$.

SODIUM SALICYLATE

The effect of sodium salicylate was examined using cartilage from three femoral heads (FH3, FH4, and
FH5) and in each case a significant depression of ${ }^{35} \mathrm{SO}_{4}$ incorporation $(\mathrm{P}<0.005)$ was observed with increasing sodium salicylate concentration up to $5.0 \mathrm{mmol} / \mathrm{l}$ (Fig. 3; Table III). As for indomethacin, the difference between individual femoral heads was highly significant $(P<0.005)$.

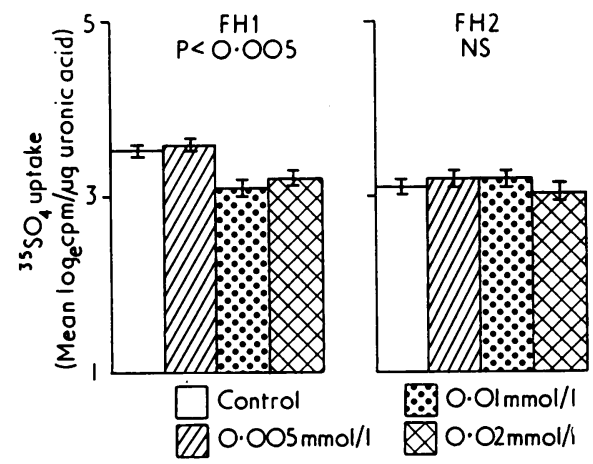

FIG. 1 Inhibition by indomethacin of ${ }^{35} \mathrm{SO}_{4}$ uptake into sulphated glycosaminoglycans of human cartilage from 2 femoral heads (FH1 and FH2) at concentrations of $0.005-0.02 \mathrm{mmol} / \mathrm{l}$. Increased drug concentration caused a highly significant $(\mathrm{P}<0.005)$ decrease in ${ }^{35} \mathrm{SO}_{4}$ uptake for FH1. The drug had no significant effect for $\mathrm{FH} 2$

Table I Analysis of variance for the effect of indomethacin $(0.005-0.02 \mathrm{mmol} / \mathrm{l})$ on sulphated glycosaminoglycan synthesis in cartilage from 2 femoral heads

\begin{tabular}{|c|c|c|c|c|}
\hline Source of variation & Sum of squares & $d f$ & Mean square & $F$ \\
\hline $\begin{array}{l}F H I \\
\text { Drug concentration (D) } \\
\text { Incubation time (T) } \\
\text { Interaction between D and } \mathrm{T} \\
\text { Residual }\end{array}$ & $\begin{array}{r}1.6818 \\
24 \cdot 0000 \\
0.9543 \\
3.0075\end{array}$ & $\begin{array}{r}3 \\
5 \\
15 \\
48\end{array}$ & $\begin{array}{l}0.5606 \\
4.8000 \\
0.0636 \\
0.0626\end{array}$ & $\begin{array}{c}8.96^{*} \\
76.68^{*} \\
1.02 \mathrm{NS}\end{array}$ \\
\hline Total & $29 \cdot 6436$ & 71 & & \\
\hline $\begin{array}{l}F H 2 \\
\text { Drug concentration (D) } \\
\text { Incubation time (T) } \\
\text { Interaction between } \mathrm{D} \text { and } \mathrm{T} \\
\text { Residual }\end{array}$ & $\begin{array}{r}0 \cdot 2283 \\
20 \cdot 2574 \\
1.9671 \\
2 \cdot 3623\end{array}$ & $\begin{array}{r}3 \\
5 \\
15 \\
48\end{array}$ & $\begin{array}{l}0.0761 \\
4.0515 \\
0.1311 \\
0.0492\end{array}$ & $\begin{array}{c}1 \cdot 55 \mathrm{NS} \\
82 \cdot 35^{*} \\
2 \cdot 66 \dagger\end{array}$ \\
\hline Total & $24 \cdot 8151$ & 71 & & \\
\hline $\begin{array}{l}\text { Pooled FHI and FH2 } \\
\text { Drug concentration (D) } \\
\text { Incubation time (T) } \\
\text { Femoral head }(\mathrm{F}) \\
\text { Interaction between } \mathrm{D} \text { and } \mathrm{T} \\
\text { Interaction between } \mathrm{D} \text { and } \mathrm{F} \\
\text { Interaction between } \mathrm{T} \text { and } \mathrm{F} \\
\text { Interaction between } \mathrm{D} \text { and } \mathrm{T} \text { and } \mathrm{F} \\
\text { Residual }\end{array}$ & $\begin{array}{r}1.0678 \\
44.0868 \\
1.6346 \\
1.2175 \\
0.8423 \\
0.6574 \\
1.2171 \\
5.3698\end{array}$ & $\begin{array}{r}3 \\
5 \\
1 \\
15 \\
3 \\
5 \\
15 \\
96\end{array}$ & $\begin{array}{c}0.3559 \\
8.8174 \\
1.6346 \\
0.0812 \\
0.2808 \\
0.1315 \\
0.0811 \\
0.0559\end{array}$ & $\begin{array}{l}1 \cdot 27 \mathrm{NS} \\
67 \cdot 05^{*} \\
29 \cdot 24^{*} \\
1.00 \mathrm{NS} \\
5 \cdot 02 \dagger \\
2 \cdot 35 \ddagger \\
1.45 \mathrm{NS}\end{array}$ \\
\hline Total & 56.0933 & 143 & & \\
\hline
\end{tabular}

In this and following tables, the significance of $F$ tests is as follows: * very highly significant $(P<0.005)$;

$\dagger$ highly significant $(\mathrm{P}<0.01) ; \ddagger$ significant $(\mathrm{P}<0.05)$; NS, not significant. 
Table II Analysis of variance for the effect of indomethacin $(0 \cdot 1-1 \cdot 0 \mathrm{mmol} / \mathrm{l})$ on sulphated glycosaminoglycan synthesis in cartilage from 3 femoral heads

\begin{tabular}{|c|c|c|c|c|}
\hline Source of variation & Sum of squares & $d f$ & Mean square & $F$ \\
\hline \multicolumn{5}{|l|}{ FH3 } \\
\hline Drug concentration (D) & 23.8747 & 3 & $7 \cdot 9582$ & $6.00 * \tau$ \\
\hline Incubation time $(\mathrm{T})$ & $5 \cdot 2911$ & 5 & 1.0582 & $0.80 \mathrm{NS}$ \\
\hline Interaction between $\mathrm{D}$ and $\mathrm{T}$ & $9 \cdot 5374$ & 15 & 0.6358 & $0.48 \mathrm{NS} \overline{\bar{G}}$ \\
\hline Residual & 63.6164 & 48 & $1 \cdot 3253$ & $\bar{\pi}$ \\
\hline Total & $102 \cdot 3196$ & 71 & & 8 \\
\hline \multicolumn{5}{|l|}{ FH4 } \\
\hline Drug concentration (D) & 3.0620 & 3 & 1.0207 & $7 \cdot 36^{*}$ \\
\hline Incubation time $(\mathrm{T})$ & $5 \cdot 5581$ & 5 & $1 \cdot 1116$ & $8.01^{*}$ \\
\hline Interaction between $\mathrm{D}$ and $\mathrm{T}$ & $12 \cdot 5315$ & 15 & 0.8354 & $6 \cdot 02^{*}$ \\
\hline Residual & 6.5548 & 48 & $0 \cdot 1387$ & \\
\hline Total & $27 \cdot 7064$ & 71 & & \\
\hline \multicolumn{5}{|l|}{ FH 5} \\
\hline Drug concentration (D) & $4 \cdot 4176$ & 3 & 1.4725 & $4 \cdot 67 \dagger$ \\
\hline Incubation time $(\mathrm{T})$ & $3 \cdot 5654$ & 5 & $0 \cdot 7131$ & $2 \cdot 26 \mathrm{NS}_{=}$ \\
\hline Interaction between $\mathrm{D}$ and $\mathrm{T}$ & $4 \cdot 8124$ & 15 & $0 \cdot 3208$ & $1 \cdot 02 \ddagger-$ \\
\hline Residual & $15 \cdot 1177$ & 48 & $0 \cdot 3150$ & a \\
\hline Total & $27 \cdot 9131$ & 71 & & 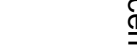 \\
\hline \multicolumn{5}{|l|}{ Pooled FH3, FH4, and FH5 } \\
\hline Drug concentration (D) & $21 \cdot 7049$ & 3 & $7 \cdot 2350$ & $7 \cdot 49 * \circ$ \\
\hline Incubation time $(\mathrm{T})$ & $5 \cdot 2774$ & 5 & $1 \cdot 0555$ & $0.37 \mathrm{~N}$ \\
\hline Femoral head $(F)$ & $26 \cdot 8188$ & 2 & $13 \cdot 4094$ & $22 \cdot 64^{*}=$ \\
\hline Interaction between $\mathrm{D}$ and $\mathrm{T}$ & 0.7798 & $1 \overline{5}$ & 0.0520 & 11.52*ํㅡㅁ \\
\hline Interaction between $\mathrm{D}$ and $\mathrm{F}$ & $17 \cdot 2758$ & 6 & $2 \cdot 8793$ & $4 \cdot 86 * \overline{2}$ \\
\hline Interaction between $\mathrm{T}$ and $\mathrm{F}$ & $9 \cdot 6561$ & 10 & 0.9656 & $1 \cdot 63 \mathrm{NS}$ \\
\hline Interaction between $\mathrm{D}$ and $\mathrm{T}$ and $\mathrm{F}$ & $17 \cdot 9681$ & 30 & 0.5989 & $1.01 \mathrm{NS}$ \\
\hline Residual & $85 \cdot 2889$ & 144 & 0.5923 & \\
\hline Total & $184 \cdot 7698$ & 215 & & \\
\hline
\end{tabular}

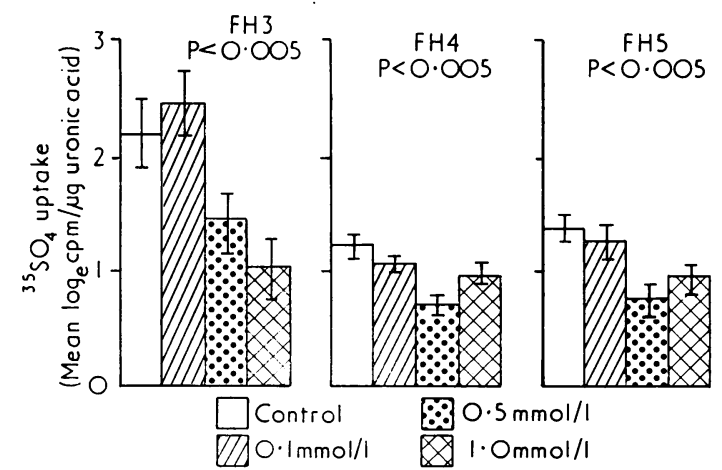

FIG. 2 Inhibition by indomethacin of ${ }^{35} \mathrm{SO}_{4}$ uptake into sulphated glycosaminoglycans of human cartilage from 3 femoral heads (FH3, FH4, and FH5) at concentrations of 0.1-1.0 mmol/l. Increased drug concentration caused a highly significant $(\mathrm{P}<0.005)$ decrease in ${ }^{35} \mathrm{SO}_{4}$ concentration in each femoral head

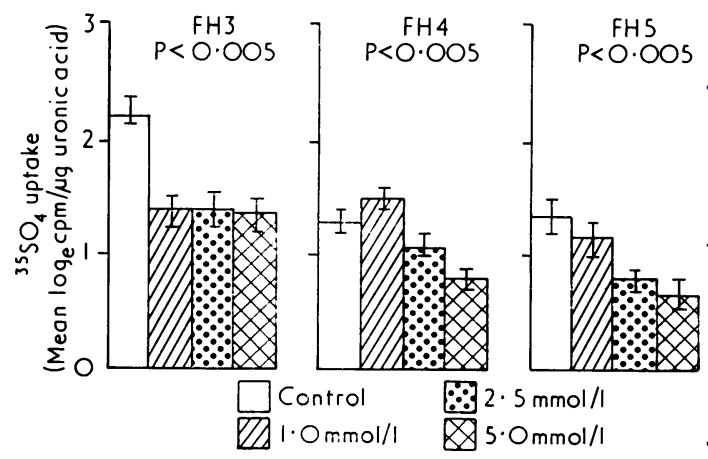

FIG. 3 Effect of sodium salicylate on ${ }^{35} \mathrm{SO}_{4}$ uptake into sulphated glycosaminoglycans of human cartilage from $3^{-}$ femoral heads (FH3, FH4, and FH5) at various concen- trations. Increased drug concentration caused a highly significant $(P<0.005)$ decrease in ${ }^{35} \mathrm{SO}_{4}$ uptake in each femoral head 
Table III Analysis of variance for the effect of sodium salicylate on sulphated glycosaminoglycan synthesis in cartilage from 3 femoral heads

\begin{tabular}{|c|c|c|c|c|}
\hline Source of variation & Sum of squares & $d f$ & Mean square & $F$ \\
\hline \multicolumn{5}{|l|}{ FH3 } \\
\hline Drug concentration (D) & 8.9590 & 3 & $2 \cdot 9863$ & $8 \cdot 01^{*}$ \\
\hline Incubation time $(\mathrm{T})$ & $12 \cdot 4122$ & 5 & $2 \cdot 4824$ & $6 \cdot 66^{*}$ \\
\hline Interaction between $\mathrm{D}$ and $\mathrm{T}$ & $9 \cdot 4279$ & 15 & 0.6285 & $1.69 \mathrm{NS}$ \\
\hline Residual & $17 \cdot 8954$ & 48 & $0 \cdot 3728$ & \\
\hline Total & $48 \cdot 6945$ & 71 & & \\
\hline \multicolumn{5}{|l|}{ FH4 } \\
\hline Drug concentration (D) & $5 \cdot 2153$ & 3 & $1 \cdot 7348$ & $12 \cdot 73 *$ \\
\hline Incubation time $(T)$ & $6 \cdot 3144$ & 5 & $1 \cdot 2629$ & $9 \cdot 25 *$ \\
\hline Interaction between $\mathrm{D}$ and $\mathrm{T}$ & 4.4988 & 15 & 0.2999 & $2 \cdot 20 \ddagger$ \\
\hline Residual & $6 \cdot 5548$ & 48 & $0 \cdot 1366$ & \\
\hline Total & $22 \cdot 5833$ & 71 & & \\
\hline \multicolumn{5}{|l|}{ FH5 } \\
\hline Drug concentration (D) & $6 \cdot 6213$ & 3 & $2 \cdot 2071$ & $8 \cdot 10^{*}$ \\
\hline Incubation time $(\mathrm{T})$ & 5.6559 & 5 & $1 \cdot 1312$ & $4 \cdot 15^{*}$ \\
\hline Interaction between $\mathrm{D}$ and $\mathrm{T}$ & $4 \cdot 3078$ & 15 & $0 \cdot 2872$ & $1.05 \mathrm{NS}$ \\
\hline Residual & 13.0825 & 48 & $0 \cdot 2726$ & \\
\hline Total & $29 \cdot 6675$ & 71 & & \\
\hline \multicolumn{5}{|l|}{ Pooled $\mathrm{FH} 3, \mathrm{FH} 4$, and $\mathrm{FH} 5$} \\
\hline Drug concentration (D) & $15 \cdot 1387$ & 3 & 5.0462 & $5 \cdot 79 \ddagger$ \\
\hline Incubation time $(\mathrm{T})$ & 20.9358 & 5 & $4 \cdot 1872$ & $2 \cdot 26 \mathrm{NS}$ \\
\hline Femoral head $(F)$ & $14 \cdot 7342$ & 2 & $7 \cdot 3671$ & $28.27 *$ \\
\hline Interaction between $\mathrm{D}$ and $\mathrm{T}$ & $7 \cdot 7244$ & 15 & 0.5150 & $1 \cdot 41 \mathrm{NS}$ \\
\hline Interaction between $\mathrm{D}$ and $\mathrm{F}$ & 3.4243 & 10 & $0 \cdot 3424$ & $12 \cdot 23^{*}$ \\
\hline Interaction between $\mathrm{T}$ and $\mathrm{F}$ & $5 \cdot 2294$ & 6 & 0.8716 & $3 \cdot 34 \dagger$ \\
\hline Interaction between $\mathrm{D}$ and $\mathrm{T}$ and $\mathrm{F}$ & 10.9675 & 30 & 0.3656 & $1 \cdot 31 \mathrm{NS}$ \\
\hline Residual & $37 \cdot 5327$ & 144 & 0.2606 & \\
\hline Total & $115 \cdot 6870$ & 215 & & \\
\hline
\end{tabular}

\section{HYDROCORTISONE}

Hydrocortisone was tested with cartilage from two femoral heads (FH3 and FH4). The effect of this steroidal anti-inflammatory drug on glycosaminoglycan synthesis differed for the two specimens. One (FH3) showed a significant inhibition of sulphate incorporation with increasing hydrocortisone concentration to $1.0 \mathrm{mmol} / 1(\mathrm{P}<0.005)$, but the other (FH4) showed no significant effect of the drug on sulphated glycosaminoglycan synthesis (Fig. 4; Table IV).

\section{BUPR OFEN}

Articular cartilage sampled from two femoral heads (FH6 and FH7) was used to investigate the effects of ibuprofen. In one experiment (FH6), ibuprofen at concentrations up to $0.2 \mathrm{mmol} / \mathrm{l}$ had no significant effect on sulphated glycosaminoglycan synthesis. In a second experiment (FH7), a highly significant depression was caused by ibuprofen at identical concentrations (Fig. 5; Table V).

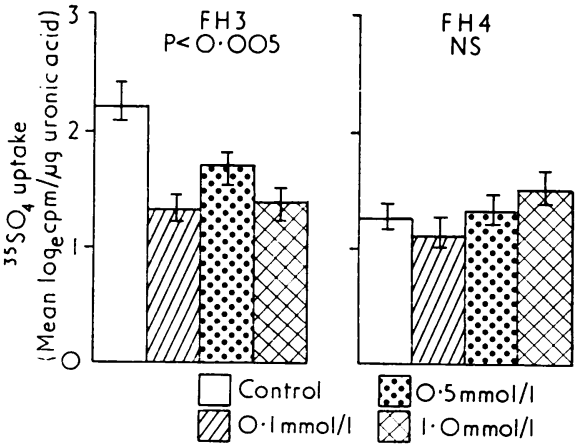

FIG. 4 Effect of hydrocortisone on ${ }^{35} \mathrm{SO}_{4}$ uptake into sulphated glycosaminoglycans of human cartilage from 2 heads (FH3 and $\mathrm{FH} 4)$ at various concentrations. Increased drug concentration caused a highly significant $(P<0.005)$ decrease in ${ }^{35} \mathrm{SO}_{4}$ uptake for $\mathrm{FH}$. The drug had no signifcant effect for $\mathrm{FH} 4$ 


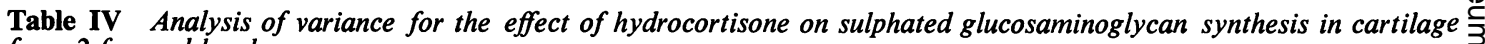
from 2 femoral heads

\begin{tabular}{|c|c|c|c|c|}
\hline Source of variation & Sum of squares & $d f$ & Mean square & $F$ \\
\hline \multicolumn{5}{|l|}{$\mathrm{FH} 3$} \\
\hline Drug concentration (D) & 8.9682 & 3 & 2.9894 & $6 \cdot 83^{*}$ \\
\hline Incubation time $(\mathrm{T})$ & $9 \cdot 6262$ & 5 & 1.9252 & $4 \cdot 40^{*}$ \\
\hline Interaction between $\mathrm{D}$ and $\mathrm{T}$ & $3 \cdot 1715$ & 15 & $0 \cdot 2114$ & $0.48 \mathrm{NS}$ \\
\hline Residual & $21 \cdot 0014$ & 48 & 0.4375 & \\
\hline Total & $42 \cdot 7673$ & 71 & & \\
\hline \multicolumn{5}{|l|}{ FH4 } \\
\hline Drug concentration (D) & $1 \cdot 5462$ & 3 & 0.5154 & $2 \cdot 55 \mathrm{NS}$ \\
\hline Incubation time $(\mathrm{T})$ & $12 \cdot 4674$ & 5 & 2.4935 & $12 \cdot 36^{*}$ \\
\hline Interaction between $\mathrm{D}$ and $\mathrm{T}$ & $5 \cdot 8657$ & 15 & $0 \cdot 3910$ & $1.94 \ddagger$ \\
\hline Residual & $9 \cdot 6846$ & 48 & $0 \cdot 2018$ & \\
\hline Total & $29 \cdot 5639$ & 71 & & \\
\hline \multicolumn{5}{|l|}{ Pooled $\mathrm{FH} 3$ and $\mathrm{FH} 4$} \\
\hline Drug concentration (D) & $4 \cdot 3049$ & 3 & 1.4350 & $0.76 \mathrm{NS}$ \\
\hline Incubation time $(T)$ & $17 \cdot 1547$ & 5 & 3.4309 & $3 \cdot 73 \mathrm{NS}$ \\
\hline Femoral head (F) & 3.5348 & 1 & $3 \cdot 5348$ & $11 \cdot 06^{*}$ \\
\hline Interaction between $\mathrm{D}$ and $\mathrm{T}$ & $7 \cdot 7960$ & 15 & 0.5197 & $20 \cdot 62 *$ \\
\hline Interaction between $\mathrm{D}$ and $\mathrm{F}$ & $5 \cdot 6503$ & 3 & 1.8834 & $5 \cdot 89 *$ \\
\hline Interaction between $\mathrm{T}$ and $\mathrm{F}$ & $4 \cdot 6040$ & 5 & 0.9208 & $2 \cdot 88 \ddagger$ \\
\hline Interaction between $\mathrm{D}$ and $\mathrm{T}$ and $\mathrm{F}$ & $0 \cdot 3775$ & 15 & 0.0252 & $12 \cdot 68^{*}$ \\
\hline Residual & $30 \cdot 6860$ & 96 & $0 \cdot 3197$ & \\
\hline Total & $74 \cdot 1082$ & 143 & & \\
\hline
\end{tabular}

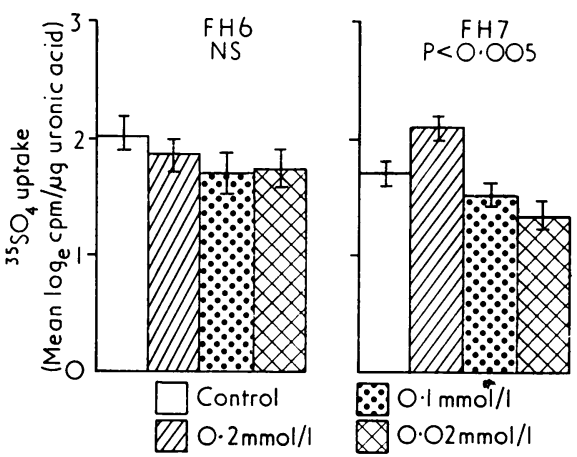

FIG. 5 Effect of ibuprofen on ${ }^{35} \mathrm{SO}_{4}$ uptake into sulphated glycosaminoglycans of human cartilage from 2 femoral heads (FH6 and FH7) at various concentrations. Increased drug concentration caused a highly significant $(\mathrm{P}<0.005)$ decrease in ${ }^{35} \mathrm{SO}_{4}$ uptake for $\mathrm{FH6}$. The drug had no significant effect for $\mathrm{FH} 7$

\section{FLURBI PROFEN}

Flurbiprofen, in contrast to the other anti-inflammatory drugs, did not depress ${ }^{35} \mathrm{SO}_{4}$ incorporation over the concentration range studied (up to 0.04 $\mathrm{mmol} / \mathrm{l})$. Its effects were examined on cartilage from two femoral heads (FH6 and FH7). One (FH6) showed that the drug had a significant effect on ${ }^{35} \mathrm{SO}_{4}$ uptake $(\mathrm{P}<0.01)$ but this was not a simple depressive effect. The lowest drug concentration
$(0.004 \mathrm{mmol} / \mathrm{l})$ produced an effect which was signil-s ficantly lower than the control; but at the two higherō concentrations $(0.02$ and $0.04 \mathrm{mmol} / \mathrm{l})$ no significantő differences from the control were observed (Fig. $\stackrel{\mathbb{Q}}{ }$ 6; Table VI). In the second femoral head (FH7), $\mathrm{a}_{\overrightarrow{0}}$ significant rise in ${ }^{35} \mathrm{SO}_{4}$ uptake was evident at 3 flurbiprofen concentrations of $0.02 \mathrm{mmol} / \mathrm{l}$, but this? response was not observed at the other drug con-0. centrations $(0.004 \mathrm{mmol} / 1$ and $0.04 \mathrm{mmol} / \mathrm{l})$ usedọ (Fig. 6).
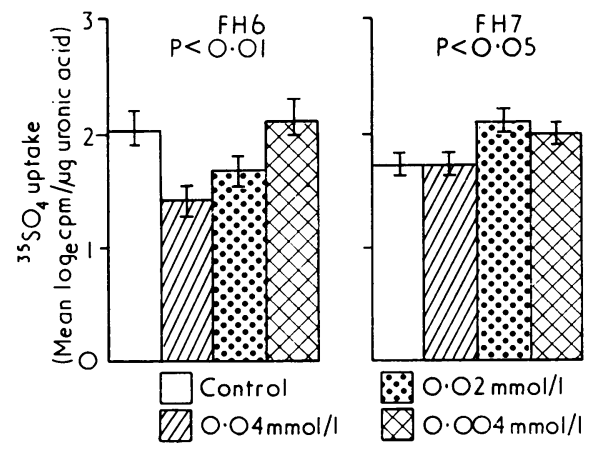

FIG. 6 Effect of flurbiprofen on ${ }^{35} \mathrm{SO}_{4}$ uptake into 0 sulphated glycosaminoglycans of human cartilage from 20. femoral heads (FH6 and $\mathrm{FH} 7$ ) at various concentrations. Inhibition of ${ }^{35} \mathrm{SO}_{4}$ uptake was not observed in either case up to the maximum concentration used 
Table $\mathbf{V}$ Analysis of variance for the effect of ibuprofen on sulphated glycosaminoglycan synthesis in cartilage from 2 femoral heads

\begin{tabular}{|c|c|c|c|c|}
\hline Source of variation & Sum of squares & $d f$ & Mean square & $F$ \\
\hline $\begin{array}{l}\text { FH6 } \\
\text { Drug concentration (D) } \\
\text { Incubation time (T) } \\
\text { Interaction between D and T } \\
\text { Residual }\end{array}$ & $\begin{array}{r}1 \cdot 3960 \\
5 \cdot 1547 \\
3 \cdot 3153 \\
21 \cdot 2326\end{array}$ & $\begin{array}{r}3 \\
5 \\
15 \\
48\end{array}$ & $\begin{array}{l}0.4653 \\
1.0309 \\
1.2210 \\
0.4423\end{array}$ & $\begin{array}{l}1.05 \mathrm{NS} \\
2.33 \mathrm{NS} \\
0.50 \mathrm{NS}\end{array}$ \\
\hline Total & 31.0986 & 71 & & \\
\hline $\begin{array}{l}\text { FH7 } \\
\text { Drug concentration (D) } \\
\text { Incubation time (T) } \\
\text { Interaction between } \mathrm{D} \text { and } \mathrm{T} \\
\text { Residual }\end{array}$ & $\begin{array}{l}5 \cdot 8623 \\
6 \cdot 6399 \\
4 \cdot 0658 \\
7 \cdot 1448\end{array}$ & $\begin{array}{r}3 \\
5 \\
15 \\
48\end{array}$ & $\begin{array}{l}1 \cdot 9541 \\
1 \cdot 3280 \\
0 \cdot 2711 \\
0 \cdot 1489\end{array}$ & $\begin{array}{c}13 \cdot 13^{*} \\
8.92^{*} \\
1.82 \mathrm{NS}\end{array}$ \\
\hline Total & $23 \cdot 7128$ & 71 & & \\
\hline $\begin{array}{l}\text { Pooled FH6 and FH7 } \\
\text { Drug concentration (D) } \\
\text { Incubation time }(\mathrm{T}) \\
\text { Femoral head }(\mathrm{F}) \\
\text { Interaction between } \mathrm{D} \text { and } \mathrm{T} \\
\text { Interaction between } \mathrm{D} \text { and } \mathrm{F} \\
\text { Interaction between } \mathrm{T} \text { and } \mathrm{F} \\
\text { Interaction between } \mathrm{D} \text { and } \mathrm{T} \text { and } \mathrm{F} \\
\text { Residual }\end{array}$ & $\begin{array}{c}4.7967 \\
10 \cdot 9684 \\
0 \cdot 08147 \\
3 \cdot 4538 \\
0 \cdot 8128 \\
2 \cdot 3772 \\
3 \cdot 9403 \\
28 \cdot 3774\end{array}$ & $\begin{array}{r}3 \\
5 \\
1 \\
15 \\
3 \\
5 \\
15 \\
96\end{array}$ & $\begin{array}{l}1.5989 \\
2 \cdot 1937 \\
0.8147 \\
0 \cdot 2303 \\
0.2709 \\
0.3962 \\
0 \cdot 2627 \\
0.2956\end{array}$ & $\begin{array}{l}4 \cdot 04 \mathrm{NS} \\
8 \cdot 10 \mathrm{NS} \\
2 \cdot 76 \mathrm{NS} \\
0 \cdot 88 \mathrm{NS} \\
0.91 \mathrm{NS} \\
1.34 \mathrm{NS} \\
0.89 \mathrm{NS}\end{array}$ \\
\hline Total & $55 \cdot 5413$ & 143 & & \\
\hline \multicolumn{5}{|c|}{$\begin{array}{l}\text { Because all variation due to interactions is nonsignificant, it can be pooled with the residual variation, and F-tests } \\
\text { recalculated as follows: }\end{array}$} \\
\hline $\begin{array}{l}\text { Drug concentration (D) } \\
\text { Incubation time (T) } \\
\text { Femoral head (F) } \\
\text { Residual }\end{array}$ & $\begin{array}{r}4 \cdot 7967 \\
10 \cdot 9684 \\
0 \cdot 8147 \\
38 \cdot 9615\end{array}$ & $\begin{array}{r}3 \\
5 \\
1 \\
134\end{array}$ & $\begin{array}{l}1 \cdot 5989 \\
2 \cdot 1937 \\
0 \cdot 8147 \\
0 \cdot 2886\end{array}$ & $\begin{array}{l}5 \cdot 45^{*} \\
7 \cdot 60^{*} \\
2 \cdot 82 \text { NS }\end{array}$ \\
\hline Total & $55 \cdot 5413$ & 143 & & \\
\hline
\end{tabular}

\section{Discussion}

These findings highlight the variable response of human articular cartilage from different individuals to the same concentrations of anti-inflammatory drugs. Maroudas, Muir, and Wingham (1969) described differences in total hexuronic content from human femoral condyles which showed no correlation with age, and Muir, Bullough, and Maroudas (1970) observed variations in collagen content of human articular cartilage from different individuals, showing that human articular cartilage is variable in both composition and metabolism. This variability, together with individual rates of drug absorption and metabolism, would account for the present findings. Previous studies on the effects of antiinflammatory drugs on glycosaminoglycan metabolism have been carried out using pure-bred laboratory animals or cell lines, which would be expected to show a more uniform response than human tissue. However, using either tissue, the conclusions reached were similar.
Our preliminary experiments on aged human articular cartilage showed that $98 \%$ of the radioactive sulphate taken up during incubation could be removed by simple washing procedures. Of the remaining radioactivity, a further $75 \%$ was removed by dialysis after papain digestion. This latter radioactivity may arise from the storage of ${ }^{35} \mathrm{SO}_{4}$ in various metabolic pools which may not necessarily be utilized in polymer synthesis at the same time (Lohmander, Antonopoulos, and Friberg, 1973; Rokosova and Bentley, 1973). A large part of this labelling may also arise from nonspecific binding with preformed matrix, since it occurs at $0^{\circ}$ in tissue which has been pre-chilled at this temperature for 30 minutes before incubation with ${ }^{35} \mathrm{SO}_{4}$, in which biosynthesis would be negligible.

In the present study, ${ }^{35} \mathrm{SO}_{4}$ incorporation into sulphated glycosaminoglycans has been defined in terms of uronic acid content of the tissue after papain digestion and dialysis. The major uronic acid containing glycosaminoglycans in articular 
Table VI Analysis of variance for the effect of flurbiprofen on sulphated glycosaminoglycan synthesis in cartilage from 2 femoral heads

\begin{tabular}{|c|c|c|c|c|}
\hline Source of variation & Sum of squares & $d f$ & Mean square & $F$ \\
\hline \multicolumn{5}{|l|}{ FH6 } \\
\hline Drug concentration (D) & 6.0994 & 3 & 2.0331 & $6 \cdot 23 \ddagger$ \\
\hline Incubation time $(\mathrm{T})$ & $11 \cdot 4515$ & 5 & $2 \cdot 2903$ & $7.02 \ddagger$ \\
\hline Interaction between $\mathrm{D}$ and $\mathrm{T}$ & 3.7097 & 15 & $0 \cdot 2473$ & $0.76 \mathrm{NS}$ \\
\hline Residual & $15 \cdot 6682$ & 48 & $0 \cdot 3264$ & \\
\hline Total & $36 \cdot 9288$ & 71 & & \\
\hline \multicolumn{5}{|l|}{$\mathrm{FH7}$} \\
\hline Drug concentration (D) & $2 \cdot 4484$ & 3 & 0.8161 & $4 \cdot 40 \ddagger$ \\
\hline Incubation time $(\mathrm{T})$ & $8 \cdot 4762$ & 5 & $1 \cdot 6952$ & $9 \cdot 14^{*}$ \\
\hline Interaction between $\mathrm{D}$ and $\mathrm{T}$ & 3.9436 & 15 & 0.2629 & $1 \cdot 42 \mathrm{NS}$ \\
\hline Residual & $8 \cdot 9072$ & 48 & $0 \cdot 1856$ & \\
\hline Total & $23 \cdot 7754$ & 71 & & \\
\hline \multicolumn{5}{|l|}{ Pooled FH6 and FH7 } \\
\hline Drug concentration (D) & 4.9118 & 3 & 1.6373 & $2 \cdot 70 \mathrm{NS}$ \\
\hline Incubation time $(\mathrm{T})$ & 18.9825 & 5 & $3 \cdot 7965$ & $12.05 \ddagger$ \\
\hline Femoral head (F) & $0 \cdot 1991$ & 1 & $0 \cdot 1991$ & $0 \cdot 78 \mathrm{NS}$ \\
\hline Interaction between $\mathrm{D}$ and $\mathrm{T}$ & $5 \cdot 0913$ & 15 & $0 \cdot 3394$ & $0.73 \mathrm{NS}$ \\
\hline Interaction between $\mathrm{D}$ and $\mathrm{F}$ & 0.9452 & 3 & $0 \cdot 3151$ & $1 \cdot 23 \mathrm{NS}$ \\
\hline Interaction between $\mathrm{T}$ and $\mathrm{F}$ & 3.6360 & 5 & $0 \cdot 6060$ & $2 \cdot 37 \ddagger$ \\
\hline Interaction between $D$ and $T$ and $F$ & 6.9525 & 15 & 0.4635 & $1.81 \ddagger$ \\
\hline Residual & $24 \cdot 5754$ & 96 & $0 \cdot 2560$ & \\
\hline Total & $65 \cdot 2938$ & 143 & & 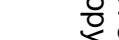 \\
\hline
\end{tabular}

cartilage are chondroitin 4 and 6 sulphates. Dermatan sulphate, hyaluronic acid, chondroitin and heparin sulphate also contain uronic acid residues, but the levels of these in articular cartilage are very small. Therefore, incorporation of ${ }^{35} \mathrm{SO}_{4}$ expressed in terms of uronic acid largely reflects the synthesis of the chondroitin sulphates. Our procedure does not take into account the incorporation of ${ }^{35} \mathrm{SO}_{4}$ into keratan sulphate. Work by Hardingham and Muir (1972) and Davidson and Small (1963) suggests, however, that the turnover rate of this component is low. Hardingham and Muir (1972), using pig laryngeal cartilage, reported that specific radioactivity measurements based on uronic acid were only slightly modified if the contribution made by keratan sulphate was accounted for. Maroudas (1975) reported preliminary results indicating that the turnover of keratan sulphate is very much slower than that of chondroitin sulphate. Her calculations of total sulphated glycosaminoglycan turnover apply, as do ours, essentially to chondroitin sulphate. Furthermore, the levels of keratan sulphate in human articular cartilage have been reported to remain relatively constant with aging after maturity (Maroudas and others, 1969; Bollet and Nance, 1966).

The reproducible sampling of the cartilage specimens was of particular importance in the present study. Full-depth samples were taken from the sur-o face to the subchondral bone, as glycosaminoglycan $\frac{0}{\Phi}$ metabolism and concentration are known to vary with depth from the surface (Jones and Lemperg, 1975; $\overrightarrow{\vec{O}}$ Maroudas, 1975; Stockwell and Scott, 1967). 3 Although Maroudas and others (1973) reported that there are only slight variations in glycosamino-? glycan content with topographical area for normal femoral heads, Bjelle (1974) did observe variations over the surfaces of femoral condyles. Therefore, 3 where possible, cartilage samples were removed from the same locality. Areas of obvious fibrillation or eburnation were excluded, but were uncommon in normal femoral heads removed after fracture.

In general, the results of our studies on the effects $\frac{7}{0}$ of anti-inflammatory drugs on the metabolism $=$ of glycosaminoglycans agree with the literature $N$ (Domenjoz, 1971; Whitehouse, 1965).

An in vivo autoradiographic study (Watson, 1976) N with rabbits receiving oral doses of indomethacin ${ }_{\sigma}^{\omega}$ showed significant suppression of ${ }^{35} \mathrm{SO}_{4}$ uptake by chondrocytes of the femoral condyles. No differences? in ${ }^{35} \mathrm{SO}_{4}$ uptake between animals receiving clinical ${ }^{\circledR}$ or higher doses of drug were observed. Our studies + were conducted over two concentration ranges, and ${ }_{0}^{-}$ the response at therapeutic drug levels (Fig. 1) was $\overparen{\mathbb{D}}$

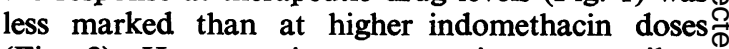
(Fig. 2). However, in our experiments, cartilage 
slices were exposed to the drug for 6 hours only, whereas Watson investigated the effects of indomethacin for periods of up to 70 days.

Roach, Tomblin, and Eyring (1975), Forney, Bentley, and Mathews (1973) and Simmons and Chrisman (1965), using morphological and histological techniques, suggested that salicylate stimulates healing of articular cartilage by some biochemical mechanism such as lysosomal stablization or enhancement of matrix formation. Salicylates and other anti-inflammatory drugs may protect cartilage by inhibiting the prostaglandin synthetase systems (Moncada, Ferreira, and Vane, 1973; Takeguchi and Sih, 1972; Tomlinson and others, 1972; Vane, 1971, 1972; Willis and others, 1972). Prostaglandins of the $\mathrm{E}$ group are now recognized as prime mediators of the inflammatory response(Glenn and others, 1972; Kaley, Massira, and Weiner, 1972; Willis and others, 1972; Kaley and Weiner, 1971; Arora, Lahiri, and Sanyal, 1970). Such a protective role would be of greater relevance in the therapy of rheumatoid arthritis than osteoarthrosis where the infiammatory component of the process is far less evident.
The ability of cartilage to function efficiently depends upon its mechanical properties which are an expression of matrix composition and organization. These in turn reflect the balance between synthesis and degradation of its components. The ability of drugs to directly or indirectly moderate joint inflammation and the associated degradative mechanisms accounts for their value in the treatment of rheumatoid arthritis, but not osteoarthrosis. Clearly, the place of some anti-inflammatory agents in the management of degenerative joint diseases should be re-examined in the light of their demonstrated inhibitory effects on cartilage glycosaminoglycan biosynthesis.

We gratefully acknowledge financial support for this project from the National Health and Medical Research Council, and The University of Sydney for a Fellowship to L. S. McK; and are grateful for the competent technical assistance of Linda Krick and Denise Greentree. We also express our gratitude to Dr. A. Underwood, Department of Biological Sciences, The University of Sydney, for his advice on the statistical methods used.

\section{References}

Arora, S., LAhiRI, P. K., AND Sanyal, R. (1970) Int. Arch. Allergy, 39, 186 (The role of prostaglandin E $_{1}$ in inflammatory process in the rat)

Benmaman, J. D., Ludoweig, J. J., AND ANDerson, C. E. (1969) Clin. Biochem., 2, 461 (Glucosamine and galactosamine distribution in human articular cartilage: relationship to age and degenerative joint disease)

BitTer, T., AND MUIR, H. (1962) Analyt. Biochem., 4, 330 (A modified uronic acid and carbazole reaction)

BJelle, A. O. (1974) Scand. J. Rheumatol., 3, 81 (Variations in content and composition of glycosaminoglycans within the articular cartilage of the lower femoral epiphysis of an adult)

- - Antonopoulos, C. A., Engfeldt, B., AND HJerteuist, S. O. (1972) Calcif. Tiss. Res., 8, 237 (Fractionation of the glycosaminoglycans of human articular cartilage on ecteola cellulose in aging and osteoarthrosis)

Bollet, A. J., AND NANCE, J. L. (1966) J. clin. Invest., 45, 1170 (Biochemical findings in normal and osteoarthritic articular cartilage. II. Chondroitin sulphate concentration and chain length, water and ash content)

- HANDY, J. R., AND STURGILl, B. C. (1963) Ibid, 42, 853 (Chondroitin sulphate concentration and proteinpolysaccharide composition of articular cartilage in osteoarthritis)

Bostrom, H. Bersten, K., ANd Whitehouse, M. W. (1964) Biochem. Pharmacol., 13, 413 (Biochemical properties of anti-inflammatory drugs. II. Some effects on sulphate $-{ }^{35} \mathrm{~S}$ metabolism in vivo)

Bullough, P., Goodfellow, J., AND O’ConNoR, J. (1973) J. Bone Jt Surg., 55A, 746 (The relationship between degenerative changes and load-bearing in the human hip)

Chalmers, I. M., Cathcart, B. J., Kumar, E. B., Dick, W. C., and Buchanan, W. W. (1972) Ann. rheum. Dis., 31, 319 (Clinico-pharmacological studies and clinical evaluation of flurbiprofen)

Collins, D. H., AND McElligotT, T. F. (1960) Ibid., 19, 318 (Sulphate uptake by chondrocytes in relation to histological changes in osteoarthritic human articular cartilage)

Curran, R. C., AND Gibson, T. (1956) Proc. roy. Soc. B., 144, 572 (The uptake of labelled sulphate by human cartilage cells and its use as a test for viability)

Davidson, E. A., ANd Small, W. (1963) Biochim. biophys. Acta, 69, 453 (Metabolism in vivo of connective tissue mucopolysaccharides. III. Chondroitin sulphate and keratosulphate of cartilage)

DomenJoz, R. (1971) 'The pharmacology of anti-rheumatic agents' in 'Rheumatoid Arthritis: Pathogenic Mechanisms and Consequences in Therapeutics', eds. W. Muller, H. G. Harwerth, and K. Fehr. Academic Press, London

ForNey, H. J., Bentley, G., AND Mathews, R. S. (1973) Orthopaedics (Oxford), 6, 19 (Salicylates and repair in adult articular cartilage)

Freeman, M. A. R. (1973) 'Mechanical failure in proteoglycan depleted cartilage' in 'Adult Articular Cartilage', p. 243. Pitman Medical, London 
Glenn, E. M., Rohloff, N., Bowman, G. J., AND Dyster, S. (1972) Arthr. and Rheum., 15, 110 (Anti-inflammatory and pro-inflammatory effects of certain prostaglandins)

HARdingham, T. E., AND MUIR, H. (1972) Biochem. J., 126, 791 (Biosynthesis of proteoglycans in cartilage slices. Fractionation by gel chromatography and equilibrium density gradient centrifugation)

Holt, L. P. J., AND Hawkins, C. F. (1965) Brit. med. J., 1, 1354 (Indomethacin: studies of absorption and of the use of indomethacin suppositories)

HuCKer, H. B., ZaCchai, A. G., Cox, S. V., Brodie, A. D., AND Coutwell, H. N. R. (1966) J. Pharmacol. exp. Ther., 153, 237 (Studies on the absorption, distribution and excretion of indomethacin in various species)

JoNeS, I. L., AND LeMPERG, R. (1975) Biochim. biophys. Acta, 392, 310 (Chondroitin sulphate of calf knee joint cartilage)

KALEY, G., AND WeINER, R. (1971) Nature New Biol., 234, 114 (Effect of prostaglandin $\mathrm{E}_{1}$ on leukocyte migration)

-, MASSIRA, E. J., AND WEINER, R. (1972) 'The role of prostaglandins in micro-circulation and inflammation' in 'Prostaglandins in Cellular Biology', eds. B. B. Pharriss and P. W. Ramwell, p. 309. Plenum Press, New York

Kalbhen, D. A., Karzel, K., and Domenjoz, R. (1967) Med. Pharmacol. Exp. 16, 185 (The inhibitory effects of some antiphlogistic drugs on the glucosamine incorporation into mucopolysaccharides synthesised by fibroblast cultures)

Karzel, K., AND Domenjoz, R. (1969) Pharmacology (Basel), 2, 302 (Actions of corticosteroids on mucopolysaccharide and protein metabolism of fibroblast monolayer cultures)

Kempson, G. E., Muir, H., Swanson, S. A. V., and Freeman, M. A. R. (1970) Biochim. biophys. Acta, 215, 70 (Correlations between stiffness and the chemical constituents of cartilage on the human femoral head)

Lohmander, S., ANtonopoulos, C. A., AND Friberg, U. (1973) Ibid., 304, 430 (Chemical and metabolic heterogeneity of chondroitin sulphate and keratan sulphate in guinea pig cartilage and nucleus pulposus)

Lust, G., AND Pronsky, W. (1972) Clin. chim. Acta, 39, 281 (Glycosaminoglycan contents of normal and degenerative articular cartilage from dogs)

McDevitt, C. A. (1973) Ann. rheum. Dis., 32, 364 (Biochemistry of articular cartilage. Nature of proteoglycans and collagen of articular cartilage and their role in aging and in osteoarthrosis)

Mankin, H. J. (1974) New Engl. J. Med., 291, 1285 (The reaction of articular cartilage to injury and osteoarthritis)

—., AND LipIEI.LO, L. (1970) J. Bone Jt Surg., 52A, 424 (Biochemical and metabolic abnormalities in articular cartilage from osteoarthritic human hips)

- - —, (1971 J. clin. Invest., 50, 1712 (The glycosaminoglycans of normal and arthritic cartilage)

- Dorfman, H., Lipiello, L., AND Zarins, A. (1971) J. Bone Jt Surg., 53A, 523 (Biochemical and metabolic abnormalities in articular cartilage from osteoarthritic human hips. II. Correlation of morphology with biochemical and metabolic data)

MaroudAs, A. (1975) Phil. Trans. B., 271, 293 (Glycosaminoglycan turnover in articular cartilage)

-, Evans, H., AND Almeida, L. (1973) Ann. rheum. Dis., 32, 1 (Cartilage of the hip joint. Topographical variation of glycosaminoglycan content in normal and fibrillated tissue)

—, MUIR, H., AND WinghaM, J. (1969) Biochim. biophys. Acta, 177, 492 (The correlation of fixed negative charge with glycosaminoglycan content of human articular cartilage)

MATTHEwS, B. F. (1953) Brit. med. J., 2, 660 (Composition of articular cartilage in osteoarthritis: changes in collagen chondroitin sulphate ratio)

Mills, R. F., Adams, S. S., Cliffe, E. E., Dickinson, W., and Nicholson, J. S. (1973) Xenobiotica, 3, 589 (The metabolism of ibuprofen)

Moncada, S., Ferreira, S. H., AND Vane, J. R. (1973) Nature, 246, 217 (Prostaglandins, aspirin-like drugs and the oedema of inflammation)

Muir, H., Bullough, P., ANd Maroudas, A. (1970) J. Bone Jt Surg., 52B, 554 (The distribution of collagen in human articular cartilage with some of its physiological implications)

Radin, E. L., Paul, I. L., AND Rose, R. M. (1975) Ann. rheum. Dis., 34, Suppl., 2 (Mechanical factors in the aetiology of osteoarthritis)

Roach, J. E., Tomblin, W., AND Eyring, E. J. (1975) Clin. Orthop., 106, 350 (Comparison of the effects of steroid, aspirin and sodium salicylate on articular cartilage)

Rokosova B., AND BentLey, J. P. (1973) Biochim. biophys. Acta, 297, 473 (The uptake of glucose $\left({ }^{14} \mathrm{C}\right.$ ) in rabbit ear cartilage proteoglycans isolated by differential extraction and by collagenase digestion)

Simmons, D. P., And Chrisman, O. D. (1965) Arthr. and Rheum., 8, 960 (Salicylate inhibition of cartilage degeneration)

SNEDECOR, G. W., AND Cochran, W. G. (1967) In 'Statistical Methods', 6th ed. Iowa State University Press

Stockwell, R. A., AND ScotT, J. E. (1967) Nature, 215, 1376 (Distribution of acidic glycosaminoglycans in human articular cartilage)

TAKeguchi, C., AND SiH, C. J. (1972) Prostaglandins, 2, 169 (A rapid spectrophotometric assay for prostaglandin synthetase: application to the study of non-steroidal anti-inflammatory agents)

Tomlinson, R. V., Ringold, H. J., QuReshi, M. C., AND ForChiflli, E. (1972) Biochem. biophys. Res. Commun., 46, 552 (Relationship between inhibition of prostaglandin synthesis and drug efficacy: support for the current theory on mode of action of aspirin-like drugs)

Trowell, O. A. (1959) Exp. Cell Res., 16, 118 (The culture of mature organs in a synthetic medium) 
VANE, J. (1971) Nature New Biol., 231, 232 (Inhibition of prostaglandin synthesis as a mechanism of action for aspirin-like drugs)

, (1972) Hosp. Pract, 7, 61 (Prostaglandins and the aspirin-like drugs)

WATSON, M. (1976) Rheum. Rehab., 15, 26 (The suppressing effect of indomethacin on articular cartilage)

WhiteHouse, M. W. (1965) Drug. res., 8, 321 (Some biochemical and pharmacological properties of antiinflammatory drugs)

—, AND Bostrom, H. (1962) Biochem. Pharmacol., 11, 1175 (The effects of some anti-inflammatory (antirheumatic) drugs on the metabolism of connective tissue)

Willis, A., Davison, P., Ramwell, P. W., Brocklehurst, W., and Smith, B. (1972) 'Release and actions of prostaglandins in inflammation and fever. Inhibition by anti-inflammatory and anti-pyretic drugs' in 'Prostaglandins in Cellular Biology', eds. B. B. Pharriss and P. W. Ramwell, p. 227. Plenum Press, New York. 